\title{
Generalized ESO and Predictive Control Based Robust Autopilot Design
}

\author{
Bhavnesh Panchal and S. E. Talole \\ Department of Aerospace Engineering, Defence Institute of Advanced Technology, Girinagar, Pune 411025, India \\ Correspondence should be addressed to S. E. Talole; setalole@hotmail.com
}

Received 5 November 2015; Revised 21 April 2016; Accepted 4 May 2016

Academic Editor: Xiao He

Copyright ( 2016 B. Panchal and S. E. Talole. This is an open access article distributed under the Creative Commons Attribution License, which permits unrestricted use, distribution, and reproduction in any medium, provided the original work is properly cited.

\begin{abstract}
A novel continuous time predictive control and generalized extended state observer (GESO) based acceleration tracking pitch autopilot design is proposed for a tail controlled, skid-to-turn tactical missile. As the dynamics of missile are significantly uncertain with mismatched uncertainty, GESO is employed to estimate the state and uncertainty in an integrated manner. The estimates are used to meet the requirement of state and to robustify the output tracking predictive controller designed for nominal system. Closed loop stability for the controller-observer structure is established. An important feature of the proposed design is that it does not require any specific information about the uncertainty. Also the predictive control design yields the feedback control gain and disturbance compensation gain simultaneously. Effectiveness of GESO in estimation of the states and uncertainties and in robustifying the predictive controller in the presence of parametric uncertainties, external disturbances, unmodeled dynamics, and measurement noise is illustrated by simulation.
\end{abstract}

\section{Introduction}

Traditionally, missile autopilots are designed using linear control techniques wherein missile dynamics are linearized around certain number of operating points in the flight envelope. Subsequently, controllers are designed at the operating points and gain scheduling is employed to obtain desired performance in the complete flight envelope. To this end, linear control approaches have dominated missile autopilot design over the past several decades [1-3]. To address the issue of robustness in the face of uncertainties in aerodynamic parameters, various robust control techniques such as $H_{\infty}$ and $\mu$ analysis $[4,5]$ have been proposed to design robust autopilots. One can also find specific theories such as control of linear parametrically varying (LPV) systems [6], eigenstructure assignment technique [7], Quantitative Feedback Theory [8], and extended-mean assignment technique for linear time-varying systems [9] employed for the design of missile autopilot.

There exist certain issues in many of the controllers that require discussion. Firstly, the robustification approaches require some knowledge, in terms of either bound of uncertainties or some characteristics of the same. In either case, lack of exact information on this count may result in either a highly conservative design or poor robustness and performance. Secondly, controllers that are based on state feedback require availability of full state vector. In the case of pitch autopilot, the state vector consists of angle of attack, pitch rate, and the fin deflection. While the pitch rate and fin deflection are measured, the angle of attack is not normally available. This necessitates a separate design for an observer to estimate it [10-12]. Lastly, due to the nonminimum phase characteristics of the acceleration dynamics, frequently controllers are designed through output redefinition, that is, using angle of attack (or combination of angle of attack and pitch rate) as output instead of the normal acceleration [9, 11, 13-17]. In such designs, usually the desired acceleration command is translated into an equivalent angle of attack command and the controller is designed to track the latter. Obviously such an approach may result in inferior performance whenever the requirements of desired acceleration are not translated accurately in terms of the desired angle of attack.

In this paper, addressing the issues, acceleration tracking pitch autopilot design for a tail controlled, roll position stabilized, skid-to-turn missile that is robust to uncertainties and external disturbances is proposed. By considering missile 
acceleration as output, an output tracking formulation of the continuous time predictive control approach $[18,19]$ is employed for the design of control law. The predictive controller, however, requires availability of accurate mathematical model of the plant. Also the controller needs complete state vector for its implementation. To address these issues, GESO $[20,21]$ is designed to estimate the effect of uncertainties and disturbances as well as the system states in an integrated manner. The estimates are used to meet the requirement of state vector as well as to robustify the predictive controller designed for nominal system. Notable features of the design are as follows. Firstly, the design offers robustness through uncertainty estimation and compensation without needing knowledge of any characteristic of the uncertainty. Next, the controller is acceleration tracking and thus the issues associated with output redefinition are overcome. Also, no separate observer design for estimation of angle of attack is needed as the GESO estimates the same along with the disturbances and uncertainties. Lastly, the predictive design yields the feedback control and disturbance compensation gains simultaneously and thus separate procedures for obtaining them are not needed. Closed loop stability of the system under the proposed controller-observer structure is established. Simulations are carried out by considering parametric uncertainties, external disturbances, unmodeled dynamics, and measurement noise to demonstrate the effectiveness of the approach and the results are presented. The continuous time predictive controller is not robust and also needs complete state vector for its implementation. The use of GESO has addressed both of these issues effectively. On the other hand, the GESO based design proposed in [20] needs design of feedback control gain and disturbance compensation gain. The predictive control offered a solution for the design of these gains simultaneously. In essence, the techniques have complemented each other to offer a more practical and viable solution. Thus, the novelty of the work lies in efficient use of continuous time predictive control approach for addressing a practical problem of missile autopilot design.

The remainder of the paper is organized as follows. Section 2 presents the missile pitch autopilot problem. In Section 3, predictive controller design for missile autopilot problem is presented. In Section 4, the GESO formulation is briefly reviewed and the GESO based predictive controller is presented. Closed loop stability results are presented in Section 5 and simulation results demonstrating the effectiveness of the proposed design are given in Section 6. Lastly, Section 7 concludes this work.

\section{Missile Autopilot Problem}

This work is concerned with pitch autopilot design for an aerodynamically tail controlled, roll position stabilized, skidto-turn tactical missile. The objective is to force a missile to track a desired lateral acceleration command generated by the outer guidance loop. The design is expected to offer robust stability and performance over its operational flight envelope.

As is well known, the equations that represent the dynamics of a missile are usually highly nonlinear [1]. Assuming that the missile is roll position stabilized, one can use the linearization procedure to obtain a linear model for the pitch plane as

$$
\begin{aligned}
\dot{\alpha}(t) & =z_{\alpha} \alpha(t)+z_{\delta} \delta(t)+q(t)+d_{\alpha}, \\
\dot{q}(t) & =m_{\alpha} \alpha(t)+m_{q} q(t)+m_{\delta} \delta(t)+d_{q}, \\
\dot{\delta}(t) & =-\frac{1}{\tau} \delta(t)+\frac{1}{\tau} \delta_{c}(t) \\
a_{z}(t) & =V\left[z_{\alpha} \alpha(t)+z_{\delta} \delta(t)\right]
\end{aligned}
$$

where $V$ is the velocity ( $\mathrm{m} / \mathrm{s}), \alpha$ is the angle of attack (rad), $q$ is the pitch rate $(\mathrm{rad} / \mathrm{s})$, and $\delta$ and $\delta_{c}(\mathrm{rad})$ are the actual and commanded fin deflections, respectively. The quantities $z_{\alpha}, z_{\delta}, m_{\alpha}, m_{q}$, and $m_{\delta}$ are the nominal values of seminondimensional aerodynamic force and moment derivatives. The terms $d_{\alpha}$ and $d_{q}$ represent the effect of uncertainties and external disturbance in the respective equation. To be specific,

$$
\begin{aligned}
& d_{\alpha}=\Delta z_{\alpha} \alpha(t)+\Delta z_{\delta} \delta(t)+d_{\alpha}^{\prime}, \\
& d_{q}=\Delta m_{\alpha} \alpha(t)+\Delta m_{q} q(t)+\Delta m_{\delta} \delta(t)+d_{q}^{\prime},
\end{aligned}
$$

where the quantity, $\Delta(\cdot)$, represents the uncertainty associated with the respective aerodynamic derivative and $d_{\alpha}^{\prime}$ and $d_{q}^{\prime}$ are the external disturbances, if there are any. The controlled output of the system is normal acceleration, $a_{z}\left(\mathrm{~m} / \mathrm{s}^{2}\right)$ as given by (2). It may be noted that the state equation (1) contains tail fin actuator dynamics approximated by a first-order lag with a time constant of $\tau$. The control input is the commanded fin deflection; that is, $u=\delta_{c}$.

In this work, as considered in many designs, it is assumed that measurements of lateral acceleration, $a_{z}(t)$, and pitch rate, $q(t)$, are available and thus the quantities form the measured outputs. Therefore, considering $a_{z}(t)$ as the controlled output and $a_{z}(t)$ and $q(t)$ as measured outputs, dynamics (1)(2) can be written in state space model form as

$$
\begin{aligned}
\dot{x}_{p} & =A_{p} x_{p}+B_{p} u+B_{d} d, \\
y & =C_{p} x_{p}, \\
y_{m} & =C_{m p} x_{p},
\end{aligned}
$$

where $x_{p} \triangleq\left[\begin{array}{lll}\alpha(t) & q(t) & \delta(t)\end{array}\right]^{T}$ is the plant state vector, $y$ is the controlled output, and $y_{m}$ is the measured output vector. The other quantities in (4) are

$$
\begin{aligned}
& A_{p}=\left[\begin{array}{ccc}
z_{\alpha} & 1 & z_{\delta} \\
m_{\alpha} & m_{q} & m_{\delta} \\
0 & 0 & -\frac{1}{\tau}
\end{array}\right] ; \\
& B_{p}=\left[\begin{array}{l}
0 \\
0 \\
\frac{1}{\tau}
\end{array}\right],
\end{aligned}
$$




$$
\begin{aligned}
C_{p} & =\left[\begin{array}{lll}
V z_{\alpha} & 0 & V z_{\delta}
\end{array}\right], \\
C_{m p} & =\left[\begin{array}{ccc}
V z_{\alpha} & 0 & V z_{\delta} \\
0 & 1 & 0
\end{array}\right], \\
B_{d} & =\left[\begin{array}{ll}
1 & 0 \\
0 & 1 \\
0 & 0
\end{array}\right] ; \\
d & =\left[\begin{array}{l}
d_{\alpha} \\
d_{q}
\end{array}\right] .
\end{aligned}
$$

From (1), it can be seen that the uncertainties are mismatched; that is, they do not appear in the control input channel and therefore compensating their effect may not be a straightforward task. In this work, GESO is used to estimate the mismatched uncertainty to robustify predictive controller.

\section{Predictive Controller Design}

The continuous time predictive control approach represents one viable approach for designing controllers for nonlinear systems. In this approach, first introduced by $\mathrm{Lu}[18,19]$, the state or output response of dynamical system is predicted by appropriate functional expansions and a quadratic performance measure based on the predicted errors in the actual response and desired response and current control expenditure is minimized pointwise to obtain an optimal feedback control law. One has state tracking problem if the desired response is of state and output tracking problem if the desired response is of output. Since then, various applications of the approach have appeared in the literature [11, 22-26].

Here, an output tracking formulation of the continuous time predictive control approach is used for the design of acceleration tracking control law. To this end, consider the system of (1)-(2). Suppose that the desired output trajectory is specified by $y^{\star}(t)$. Given the present output, $y(t)$, at any instant $t$, the current control $u(t)$ determines the output response in the immediate future. To predict the output response due to present control input, the following procedure is followed. In doing so, certain assumptions have been made. Firstly, the contribution of control surfaces in the force equation has been neglected as the control surfaces are primarily moment producing devices. As is well known, the control surfaces are essentially moment producing devices. The surfaces generate small control force; however, since they are usually located far away from the center of gravity of the missile, they generate significant moments. Since the magnitude of the control forces is significantly smaller comparative to the forces generated by the missile due to angle of attack, they are usually neglected in controller design. The assumption has been routinely used as can be seen from $[14,17,27]$. Mathematically, the assumption results in relative degree of the controlled output, $y(t)$, as three. Next, it is assumed that the rate of change of the uncertainties, that is, $\dot{d}_{\alpha}$ and $\dot{d}_{q}$, is negligible. Now following the output tracking formulation of predictive controller, the output at time $(t+h)$ is predicted by approximating $y(t+h)$ by 3rd-order Taylor series at $t$. Thus, one can express $y(t+h)$ as a function of $u(t)$ as

$$
y(t+h) \approx y(t)+h \dot{y}(t)+\frac{h^{2}}{2} \ddot{y}(t)+\frac{h^{3}}{6} \ddot{y}(t),
$$

where $h>0$ is designated as the prediction horizon and

$$
\begin{aligned}
& y=a_{z}(t) \\
& \dot{y} \approx V\left[z_{\alpha}^{2} \alpha(t)+z_{\alpha} q(t)+z_{\alpha} d_{\alpha c}\right],
\end{aligned}
$$

where $d_{\alpha c}=d_{\alpha}+z_{\delta} \delta$. Note that, by defining the uncertainty in this manner, the neglected control forces are accounted for in the design. In similar manner, higher-order output derivatives are obtained as

$$
\begin{aligned}
\ddot{y} \approx & V\left[\left(z_{\alpha}^{3}+z_{\alpha} m_{\alpha}\right) \alpha(t)+\left(z_{\alpha}^{2}+z_{\alpha} m_{q}\right) q(t)\right. \\
& \left.+z_{\alpha} m_{\delta} \delta(t)+z_{\alpha}^{2} d_{\alpha c}+z_{\alpha} d_{q}\right] \\
\ddot{y} \approx & V\left\{\left(z_{\alpha}^{4}+2 z_{\alpha}^{2} m_{\alpha}+z_{\alpha} m_{q} m_{\alpha}\right) \alpha(t)\right. \\
& +\left(z_{\alpha}^{3}+z_{\alpha} m_{\alpha}+m_{q} z_{\alpha}^{2}+z_{\alpha} m_{q}^{2}\right) q(t) \\
& +\left(m_{\delta} z_{\alpha}^{2}+z_{\alpha} m_{q} m_{\delta}-\frac{z_{\alpha} m_{\delta}}{\tau}\right) \delta(t) \\
& \left.+\left(z_{\alpha}^{3}+z_{\alpha} m_{\alpha}\right) d_{\alpha c}+\left(z_{\alpha}^{2}+z_{\alpha} m_{q}\right) d_{q}+\frac{z_{\alpha} m_{\delta}}{\tau} u(t)\right\} \\
& \triangleq \Phi_{x_{p}}+\Phi_{u} u(t),
\end{aligned}
$$

where the functions $\Phi_{x_{p}}$ and $\Phi_{u}$ are defined appropriately for the sake of brevity. It may be noted that, in (9), the uncertainties $d_{\alpha c}$ and $d_{q}$ are present through $\dot{\alpha}$ and $\dot{q}$ and therefore form part of the function $\Phi_{x_{p}}$. Next, expanding the reference output $y^{\star}(t+h)$ in the 3rd-order Taylor's series yields

$$
y^{\star}(t+h) \approx y^{\star}(t)+h \dot{y}^{\star}(t)+\frac{h^{2}}{2} \ddot{y}^{\star}(t)+\frac{h^{3}}{6} \ddot{y}^{\star}(t) .
$$

In order to find the current control, $u(t)$, which improves tracking accuracy at next instant, consider a pointwise minimization of the performance index that penalizes the output tracking error at $(t+h)$ and current control expenditure $u(t)$ as

$$
J=\frac{1}{2} Q e_{c}^{2}(t+h)+\frac{1}{2} R u^{2}(t),
$$

where $e_{c}(t+h)=y(t+h)-y^{\star}(t+h)$ is the predicted output tracking error and $Q>0$ and $R \geq 0$ are the scalar weightings on predicted tracking error and current control input, respectively. The control that minimizes the 
performance measure assuming no control saturation is obtained by setting $\partial J / \partial u$ equal to zero as

$$
\begin{aligned}
& u(t)=-\frac{Q\left(h^{3} / 6\right) \Phi_{u}}{Q\left(h^{3} / 6\right)^{2} \Phi_{u}^{2}+R}\left(e_{c}+h \dot{e}_{c}+\frac{h^{2}}{2} \ddot{e}_{c}\right. \\
& \left.+\frac{h^{3}}{6}\left(\Phi_{x_{p}}-\dddot{y}^{\star}\right)\right),
\end{aligned}
$$

where $e_{c}(t)=y(t)-y^{\star}(t)=a_{z}(t)-a_{z}^{\star}(t)$. The quantities $\dot{e}_{c}(t)=\dot{y}(t)-\dot{y}^{\star}(t)$ and $\ddot{e}_{c}(t)=\ddot{y}(t)-\ddot{y}^{\star}(t)$ are evaluated using $\dot{y}, \ddot{y}$ given in (8)-(9). Controller (12) requires selection of design parameters, that is, the weightings $Q$ and $R$ and the prediction horizon, $h$, and proper choice of them is vital for achieving desired performance. It can be verified that when there is no penalty on control input, that is, for $R=0$, substitution of (12) in (9) yields the output tracking error dynamics as

$$
\ddot{e}_{c}+\frac{3}{h} \ddot{e}_{c}+\frac{6}{h^{2}} \dot{e}_{c}+\frac{6}{h^{3}} e_{c}=0
$$

from where it is obvious that asymptotic tracking of the desired output is achieved for $h>0$.

As can be seen, the predictive controller (12) requires accurate mathematical model of the plant and therefore may not offer satisfactory performance in the presence of uncertainties and external disturbances. Also, the controller needs complete state vector as well as knowledge of the uncertainties $d_{\alpha}$ and $d_{q}$ for its implementation. Addressing these issues for integral-chain nonlinear systems, standard ESO has been used for robustification of the predictive controller in [28]. In this work, as the pitch dynamics represent nonintegral-chain system with mismatched uncertainty, GESO is used for robustification of the predictive controller as discussed in the next section.

\section{Generalized ESO Based Pitch Autopilot Design}

One approach to robustify a given controller in the presence of uncertainties without requiring any knowledge of the same is through uncertainty and disturbance estimation [21]. The idea is to estimate the effect of uncertainties and disturbances and to use the opposite of it in the controller to compensate its effect. To this end, various techniques have been proposed in literature to estimate the effect of uncertainties and disturbances [15, 29-35]. In the context of the present problem, while one can use any disturbance estimator/observer to address the issue of robustness, a separate design of state observer will be required as the predictive controller needs complete state vector. An extended state observer (ESO) [3639] is one approach that meets both of these requirements simultaneously. Owing to its efficacy, a variety of applications of the ESO based control strategies, widely referred to as Active Disturbance Rejection Control, have appeared in the literature [17, 40-46].

While the standard ESO has offered viable solution to many practical problems as stated above, it needs dynamics in integral-chain system form with matched uncertainty. Extending the usefulness of the standard ESO for general systems, that is, for nonintegral-chain systems having mismatched uncertainty, a formulation of generalized ESO is proposed in [20]. The formulation is designated as generalized ESO for obvious reason. As the pitch dynamics of (1) are not in integral-chain form and do not have matched uncertainty, the GESO is employed in this work to estimate the states and uncertainty. The design, therefore, does not require coordinate transformation as done in $[17,43,46]$.

4.1. Generalized ESO. As stated in the last section, the GESO extends the functionality and utility of the standard ESO for general systems. Consequent to its appearance in [20], applications of the GESO based designs have also appeared in literature. For example, an angle of attack tracking controller for tactical missile is presented in [13], wherein the authors have employed equivalent input disturbance while designing the GESO. In the present work, an acceleration tracking controller is proposed based on the output tracking formulation of continuous time predictive control. In the design of GESO, actual disturbances are considered. One important feature of the proposed predictive design is that it yields the feedback control and disturbance compensation gains simultaneously and thus separate procedures for their designs are avoided.

Now consider the dynamics of (4). Following the similar treatment as done in standard ESO, the plant state vector is augmented by defining the uncertainties as additional states. To this end, defining $x_{4}=d_{\alpha}$ and $x_{5}=d_{q}$, the extended order system can be written as

$$
\begin{aligned}
\dot{x} & =A x+B u+E h_{d}, \\
y_{m} & =C_{m} x
\end{aligned}
$$

where

$$
\begin{aligned}
x & =\left[\begin{array}{l}
x_{p} \\
x_{4} \\
x_{5}
\end{array}\right] ; \\
A & =\left[\begin{array}{cc}
A_{p} & B_{d} \\
0_{2 \times 3} & 0_{2 \times 2}
\end{array}\right] ; \\
B & =\left[\begin{array}{c}
B_{p} \\
0_{2 \times 1}
\end{array}\right], \\
C_{m} & =\left[\begin{array}{ll}
C_{m p} & 0_{2 \times 2}
\end{array}\right] ; \\
E & =\left[\begin{array}{l}
0_{3 \times 2} \\
I_{2 \times 2}
\end{array}\right] ; \\
h_{d} & =\left[\begin{array}{l}
h_{d 1} \\
h_{d 2}
\end{array}\right],
\end{aligned}
$$

where $I$ is an identity matrix and $h_{d 1}$ and $h_{d 2}$ are the rate of change of disturbances $d_{\alpha}$ and $d_{q}$, respectively. The quantities $h_{d 1}$ and $h_{d 2}$ are assumed as unknown but bounded 


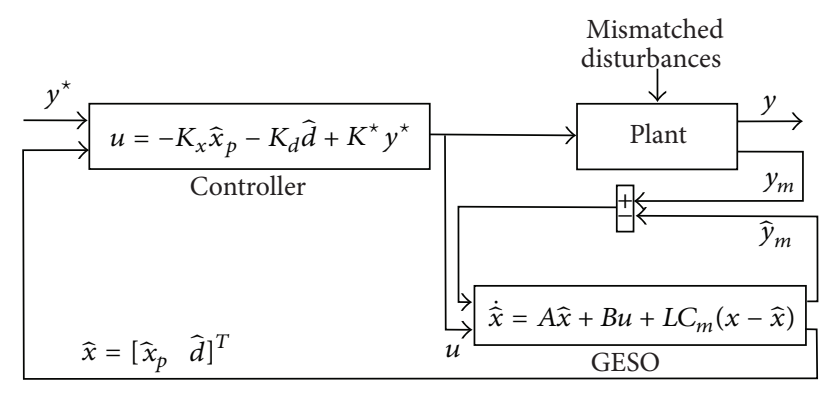

FIGURE 1: Functional block diagram of GESO based pitch autopilot.

quantities in this work. As $\left(A, C_{m}\right)$ is observable, a GESO of the following form is designed for the system of (14) as

$$
\begin{aligned}
\dot{\hat{x}} & =A \hat{x}+B u+L C_{m}(x-\hat{x}), \\
\hat{y}_{m} & =C_{m} \hat{x},
\end{aligned}
$$

where $L$ is the observer gain vector. As the uncertainties form part of the states of the extended order system, the GESO provides estimate of the states as well as the uncertainties simultaneously.

4.2. GESO Based Predictive Controller. Using the GESO estimated states and disturbances in (12), the controller takes the form as

$$
\begin{aligned}
u= & -\frac{Q\left(h^{3} / 6\right) \Phi_{u}}{Q\left(h^{3} / 6\right)^{2} \Phi_{u}^{2}+R}\left(\widehat{e}_{c}+h \dot{\vec{e}}_{c}+\frac{h^{2}}{2} \ddot{\vec{e}}_{c}\right. \\
& \left.+\frac{h^{3}}{6}\left(\widehat{\Phi}_{x_{p}}-\ddot{y}^{\star}\right)\right),
\end{aligned}
$$

where the quantities $\dot{y}, \ddot{y}$, and $\ddot{y}$ in (8) and (9) are evaluated using the estimated states and disturbances. The controller will be referred to as the GESO based predictive controller and is now implementable. The functional block diagram of the proposed pitch autopilot design is shown in Figure 1.

\section{Closed Loop Stability}

Following [20, 28, 43], closed loop stability of plant (4) with controller (17) is analyzed in this section. For simplicity, the results are worked out for set point tracking problem by treating $\dot{y}^{\star}(t), \ddot{y}^{\star}(t)$, and $\ddot{y}^{\star}(t)$ as zero. Further, one has $y^{\star}(t+$ $h)=y^{\star}(t)$ for a set point tracking problem. Now rewrite the predictive controller (12) using matrix notations of (4) as

$$
\begin{gathered}
u(t)=-\frac{Q P}{Q P^{2}+R}\left\{\left(y+h C_{\mathrm{pr}}\left(A_{\mathrm{pr}} x_{p}+B_{d} d\right)\right.\right. \\
+\frac{h^{2}}{2} C_{\mathrm{pr}}\left(A_{\mathrm{pr}}^{2} x_{p}+A_{\mathrm{pr}} B_{d} d\right) \\
\left.\left.+\frac{h^{3}}{6} C_{\mathrm{pr}}\left(A_{\mathrm{pr}}^{3} x_{p}+A_{\mathrm{pr}}^{2} B_{d} d\right)\right)-y^{\star}(t)\right\},
\end{gathered}
$$

where $P \triangleq\left(h^{3} / 6\right) C_{\mathrm{pr}} A_{\mathrm{pr}}^{2} B_{p}$. As stated earlier, since the contribution of control forces, that is, $z_{\delta} \delta$, is ignored in controller design, $A_{\mathrm{pr}}$ and $C_{\mathrm{pr}}$ are obtained from $A_{p}$ and $C_{p}$ by taking $z_{\delta}=0$. Next (18) can be written compactly as

$$
u(t)=-K_{x} x_{p}-K_{d} d+K^{\star} y^{\star},
$$

where

$$
\begin{aligned}
K_{x} & =\frac{Q P}{Q P^{2}+R}\left(C_{\mathrm{pr}}+h C_{\mathrm{pr}} A_{\mathrm{pr}}+\frac{h^{2}}{2} C_{\mathrm{pr}} A_{\mathrm{pr}}^{2}\right. \\
& \left.+\frac{h^{3}}{6} C_{\mathrm{pr}} A_{\mathrm{pr}}^{3}\right) \\
K_{d} & =\frac{\mathrm{Q} P}{Q P^{2}+R}\left(h C_{\mathrm{pr}} B_{d}+\frac{h^{2}}{2} C_{\mathrm{pr}} A_{\mathrm{pr}} B_{d}\right. \\
& \left.+\frac{h^{3}}{6} C_{\mathrm{pr}} A_{\mathrm{pr}}^{2} B_{d}\right), \\
K^{\star} & =\frac{Q P}{Q P^{2}+R} .
\end{aligned}
$$

From (20), it is obvious that the proposed predictive design yields the feedback control gain, $K_{x}$, and disturbance compensation gain, $K_{d}$, simultaneously and therefore separate procedures for their design are not needed. It can be verified that the rank condition stated in [20] for the design of $K_{d}$, that is,

$$
\begin{aligned}
& \operatorname{rank}\left(C_{\mathrm{pr}}\left(A_{\mathrm{pr}}+B_{p} K_{x}\right)^{-1} B_{p}\right) \\
& =\operatorname{rank}\left[C_{\mathrm{pr}}\left(A_{\mathrm{pr}}+B_{p} K_{x}\right)^{-1} B_{p},-C_{\mathrm{pr}}\left(A_{\mathrm{pr}}+B_{p} K_{x}\right)^{-1} B_{d}\right],
\end{aligned}
$$

is satisfied in the proposed design. Using the GESO estimates, controller (19) takes the form as

$$
u=-K_{x} \widehat{x}_{p}-K_{d} \widehat{d}+K^{\star} y^{\star} .
$$

The GESO estimation error dynamics can be obtained by subtracting (16) from (14) as

$$
\dot{e}=\left(A-L C_{m}\right) e+E h_{d},
$$

where $e$ is estimation error vector; that is, $e=\left[\begin{array}{ll}e_{p} & e_{d}\end{array}\right]^{T}$, where $e_{p}=x_{p}-\hat{x}_{p}$ and $e_{d}=d-\widehat{d}$. Using the state equation of (4) and (22) gives

$$
\dot{x}_{p}=\left(A_{p}-B_{p} K_{x}\right) x_{p}+B_{p}\left[\begin{array}{ll}
K_{x} & \left.K_{d}\right] e+h_{c}
\end{array}\right.
$$

where $h_{c}=-\left(B_{p} K_{d}\right) d+B_{d} d+B_{p} K^{\star} y^{\star}$. The output tracking error dynamics are defined as $\dot{e}_{y}=C_{p}\left(\dot{x}_{p}-\dot{x}_{p}^{\star}\right)$. Noting that $\dot{x}_{p}^{\star}=0$ for set point tracking, stability of output tracking error dynamics, $\dot{e}_{y}$, is decided by the state dynamics. Therefore, combining (23) and (24) gives

$$
\begin{aligned}
{\left[\begin{array}{c}
\dot{x}_{p} \\
\dot{e}
\end{array}\right]=} & {\left[\begin{array}{cc}
\left(A_{p}-B_{p} K_{x}\right) & B_{p}\left[K_{x} K_{d}\right. \\
0 & \left(A-L C_{m}\right)
\end{array}\right]\left[\begin{array}{c}
x_{p} \\
e
\end{array}\right] } \\
& +\left[\begin{array}{ll}
1 & 0 \\
0 & E
\end{array}\right]\left[\begin{array}{l}
h_{c} \\
h_{d}
\end{array}\right] .
\end{aligned}
$$




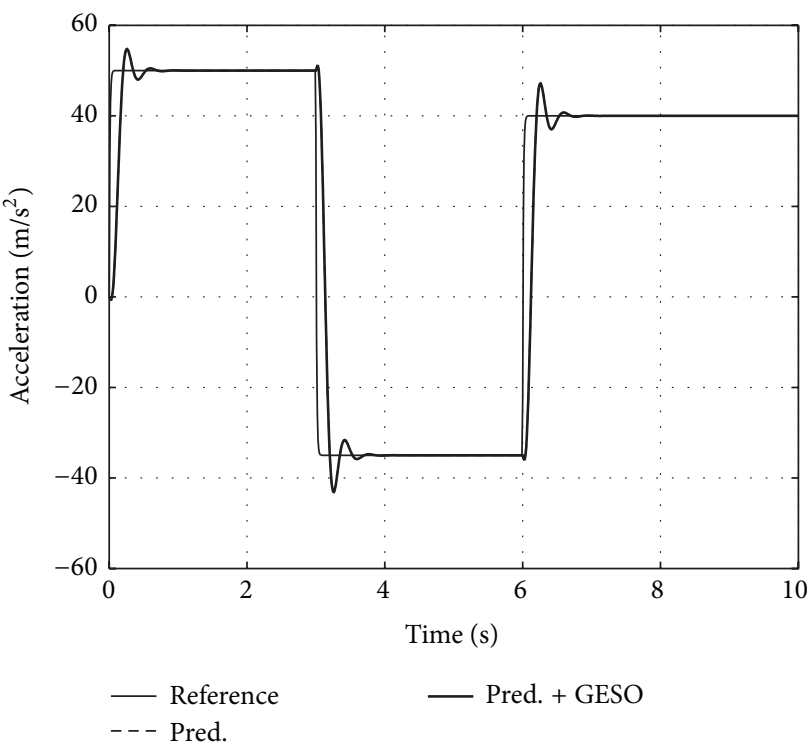

(a)

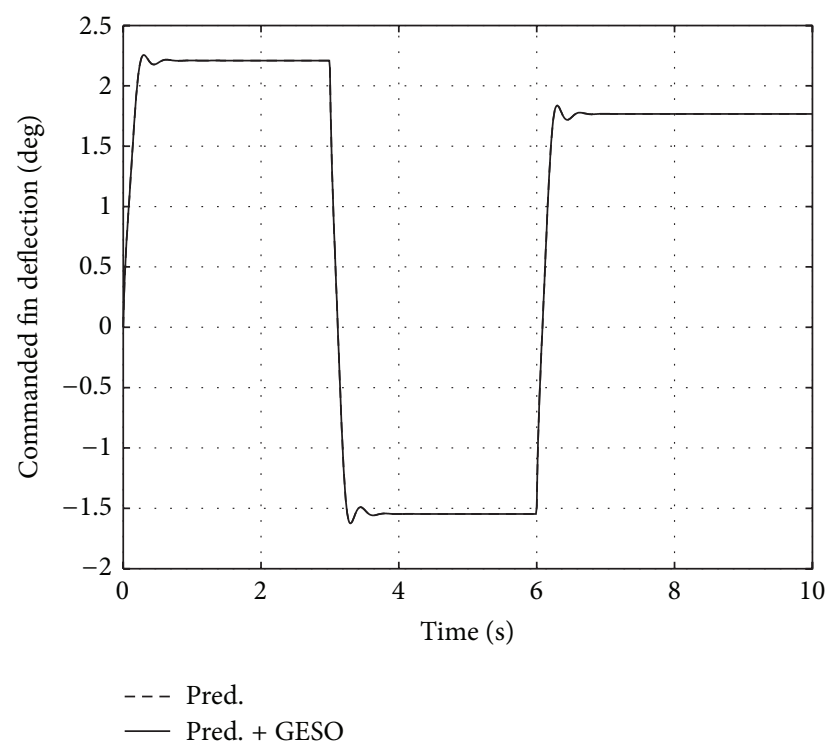

(b)

FIGURE 2: Performance in absence of uncertainties.

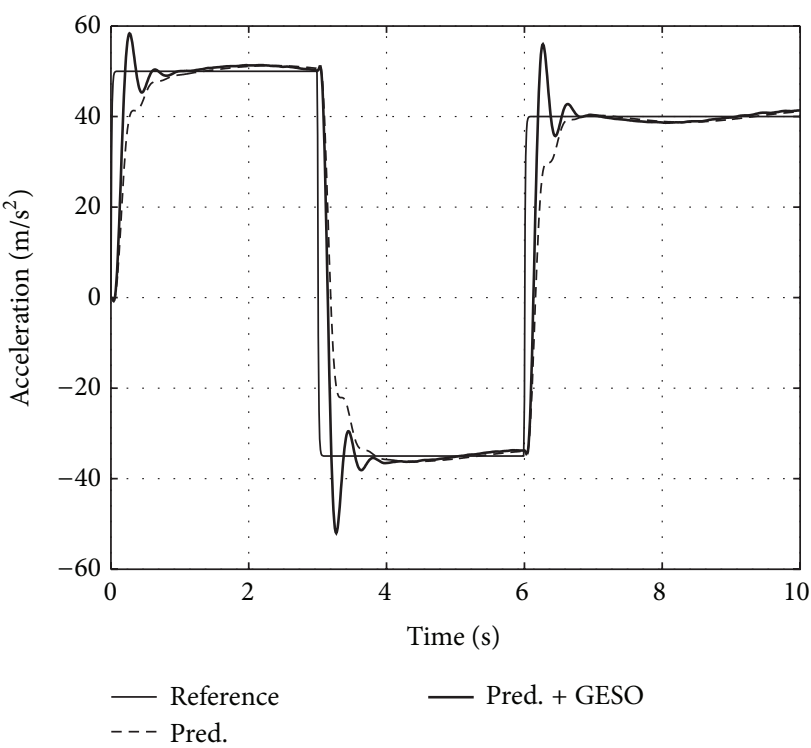

(a)

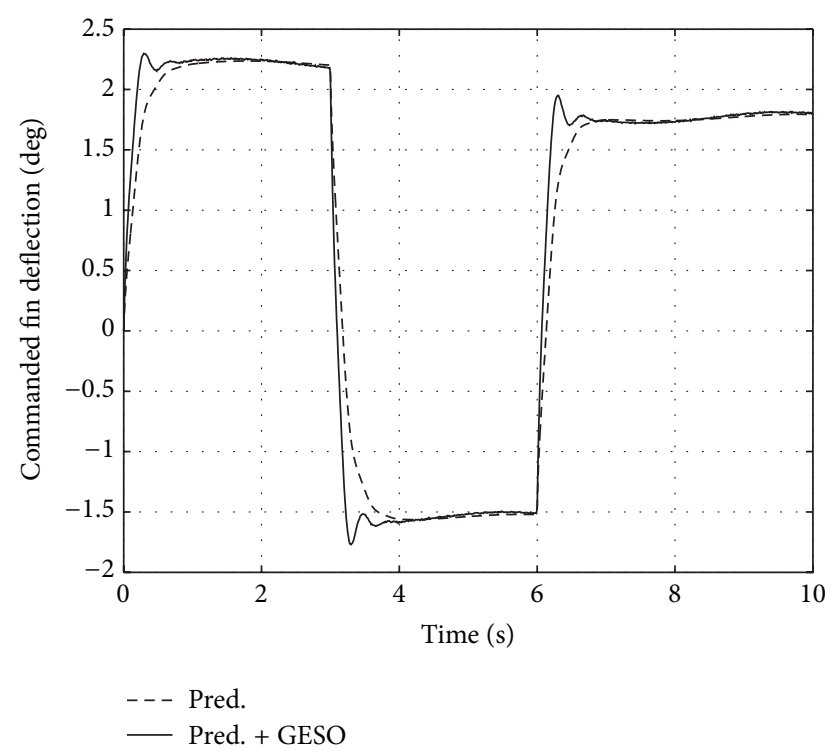

(b)

FIGURE 3: Tracking performance of GESO based predictive controller.

From (25), it can be verified that the eigenvalues of the system matrix of the closed loop error dynamics are given by the eigenvalues of $\left(A_{p}-B_{p} K_{x}\right)$ and $\left(A-L C_{m}\right)$. Since $\left(A_{p}, B_{p}\right)$ is controllable and $\left(A, C_{m}\right)$ is observable, stability of $(25)$ can be ensured through appropriate choice of the controller design parameters and the observer gain. Further, since (25) is stable, it is obvious that, under the assumption of boundedness of $h_{d}$ and $h_{c}$, bounded input-bounded output stability for linear system (25) is assured.

As is obvious from (25), the stability of the closed loop error dynamics is governed by the eigenvalues of $\left(A_{p}-B_{p} K_{x}\right)$ and $\left(A-L C_{m}\right)$ matrices. Thus, the controller gain, $K_{x}$, and the observer gain, $L$, need to be chosen appropriately to ensure stability. The observer gain depends on the observer bandwidth and therefore it can be straightforwardly obtained using the pole placement technique. The controller gain, on the other hand, depends on the predictive controller design parameters, that is, on the weightings $Q$ and $R$ and the prediction horizon $h$ as can be seen from (20). In general, it is possible to have many sets of these parameters to meet the given controller time constant or bandwidth requirement. A special case occurs when $R=0$, wherein the stability of the output tracking error dynamics is solely governed by the choice of prediction horizon as can be seen from (13). In this 


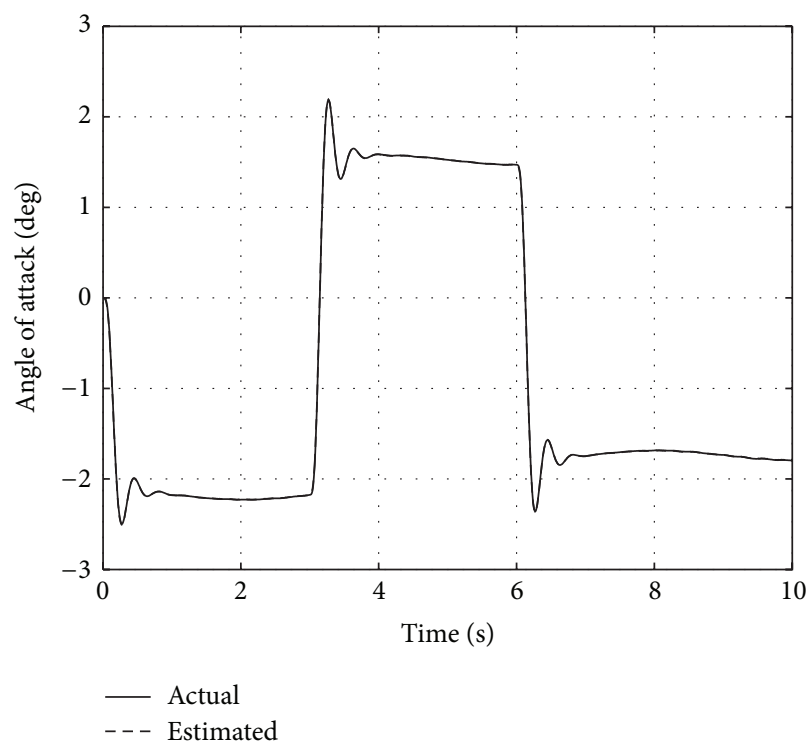

(a)

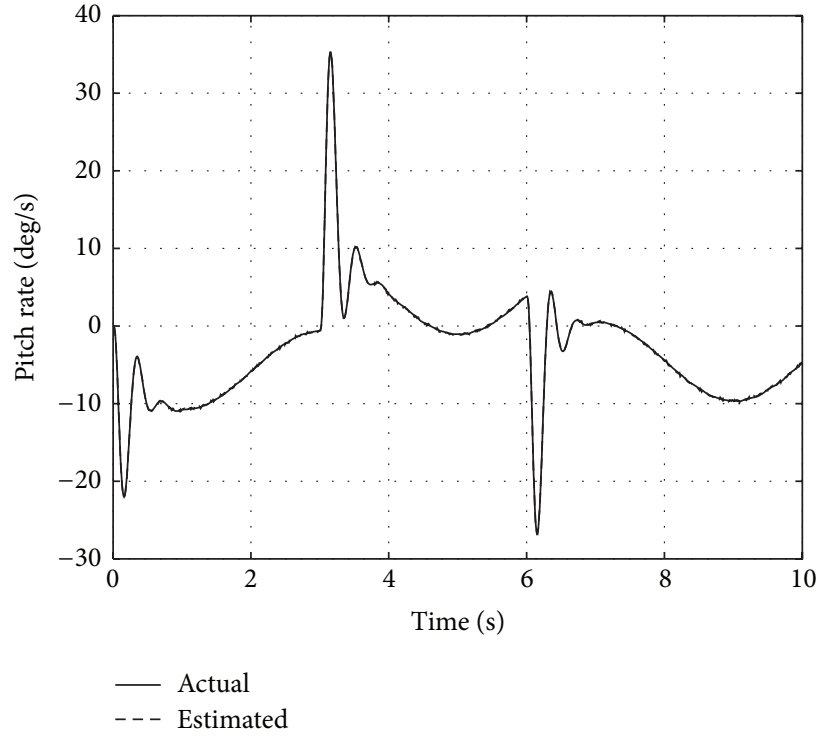

(b)

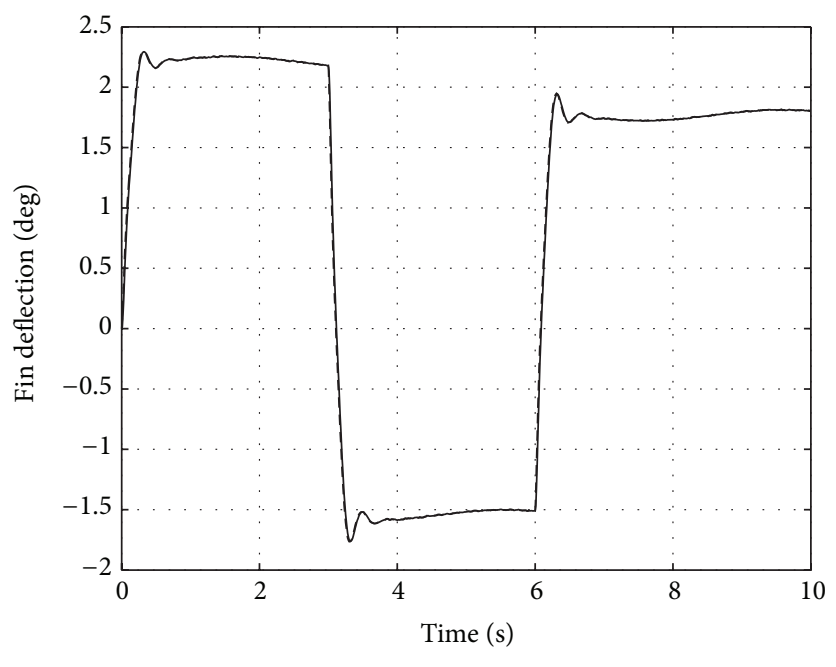

- Actual

- - - Estimated

(c)

FIgURE 4: Performance of GESO in state estimation.

case, it can be seen that the tracking dynamics are stable for $h>0$.

Lastly, it may be noted that, in this work, the rate of change of uncertainties is assumed to be negligible. Although the assumption appears to be restrictive, in practice it holds true to a great extent. It can be seen from (23) that if the rate of change of uncertainties is negligible, the GESO state estimation error dynamics exhibit asymptotic stability. However, if the uncertainties are fast varying, it is possible to tackle the same using higher-order observer. For example, if uncertainty is fast varying and its $r$ th derivative is small enough, then one can extend the order of GESO by $r$ instead of one.

\section{Simulations and Results}

Simulations are carried out to verify the performance of the GESO in state and uncertainties estimation and tracking performance of the proposed design. To this end, the nominal values of the various aerodynamic parameters for a representative tail controlled missile flying at $500 \mathrm{~m} / \mathrm{s}$ as taken from [1] are

$$
\begin{aligned}
z_{\alpha} & =-3, \\
z_{\delta} & =-0.36, \\
m_{\alpha} & =-500,
\end{aligned}
$$




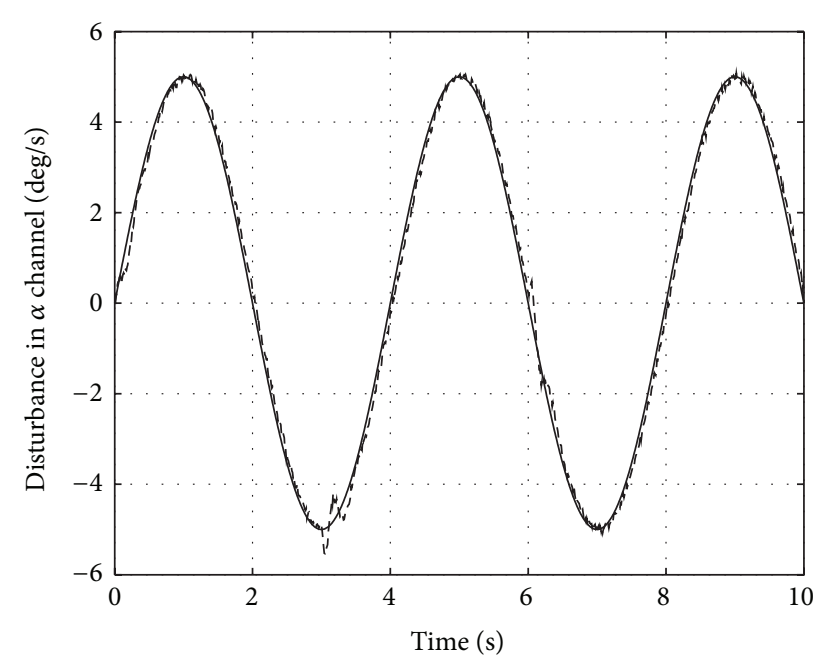

- Actual

- - - Estimated

(a)

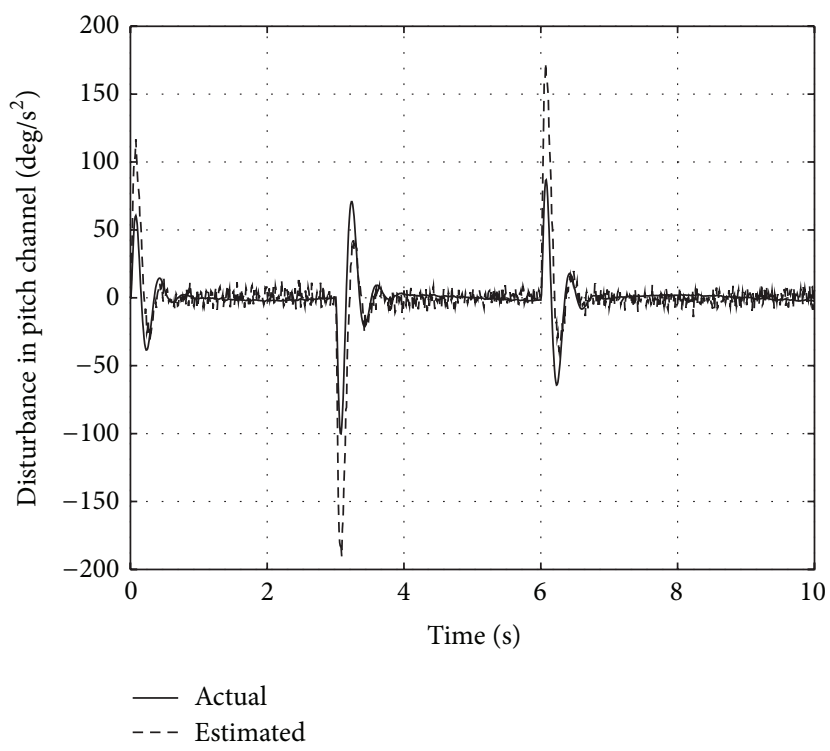

(b)

FIgURE 5: Performance of GESO in uncertainty estimation.

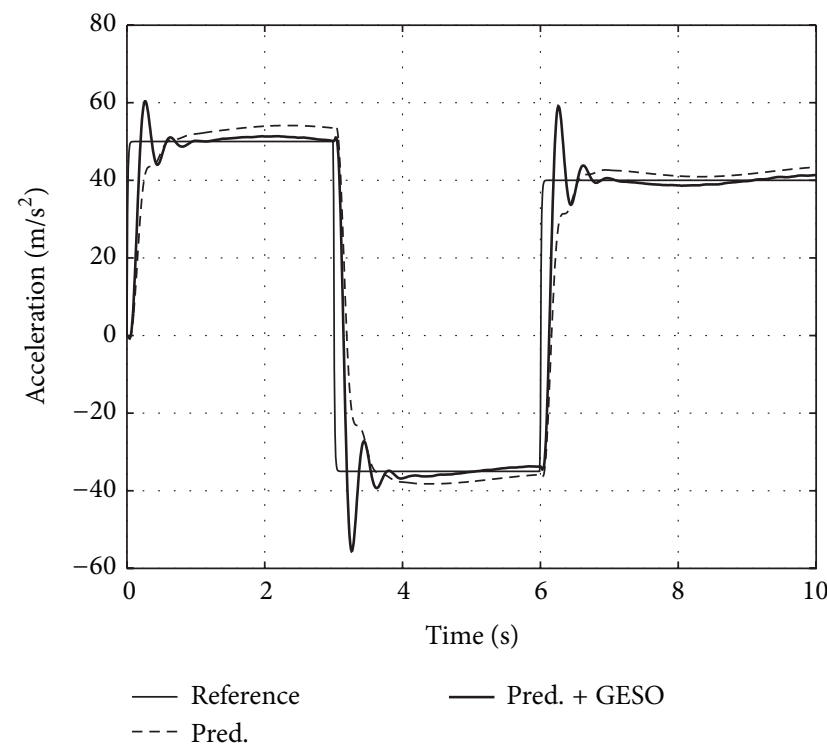

(a)

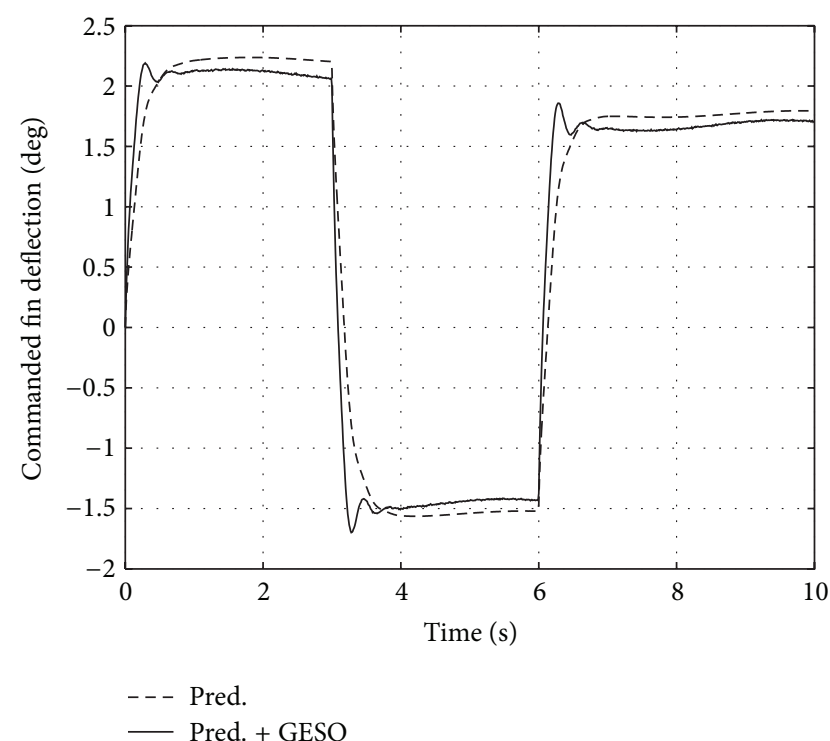

(b)

FIGURE 6: Tracking performance of GESO based predictive controller with parametric perturbation in output channel.

$$
\begin{aligned}
& m_{q}=-3, \\
& m_{\delta}=-500 .
\end{aligned}
$$

The servo time constant is taken as $\tau=0.01$. For the autopilot design, it is desired to have the closed loop time constant as 0.1 seconds. As has been shown in [19], closed loop time constant in predictive controller is of the order of prediction horizon. Therefore, prediction horizon, $h$, is taken as 0.1 . To make the observer fast enough, the observer poles are placed at -90 ; thus while the controller bandwidth is of the order of $10 \mathrm{rad} / \mathrm{s}$, the observer bandwidth is $90 \mathrm{rad} / \mathrm{s}$. The weightings $Q$ and $R$ are taken as 1 and zero, respectively. The initial conditions for plant as well as GESO are taken as zero. The reference input considered is a series of commanded acceleration in $\mathrm{m} / \mathrm{s}^{2}$ as $y^{\star}=50$ for $0<t \leq 3 \mathrm{~s}, y^{\star}=-35$ for $3<t \leq 6 \mathrm{~s}$, and $y^{\star}=40$ for $t>6 \mathrm{~s}$. In the simulations, the derivatives of reference signal required in the predictive controller (12) and GESO based predictive controller of (17) are taken as zero as the considered reference is a sort of square wave signal. With these data, simulations are carried out using controllers 


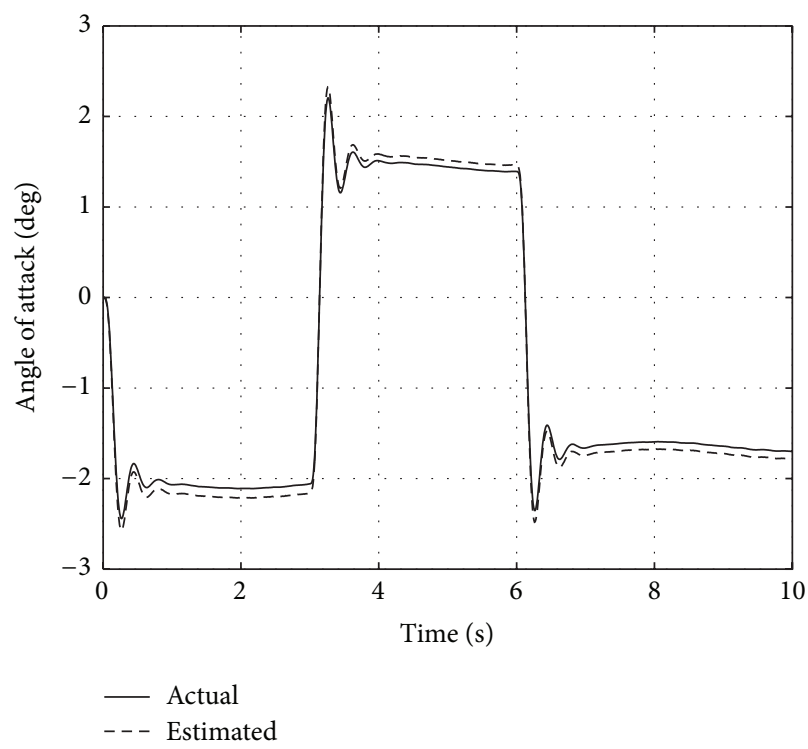

(a)

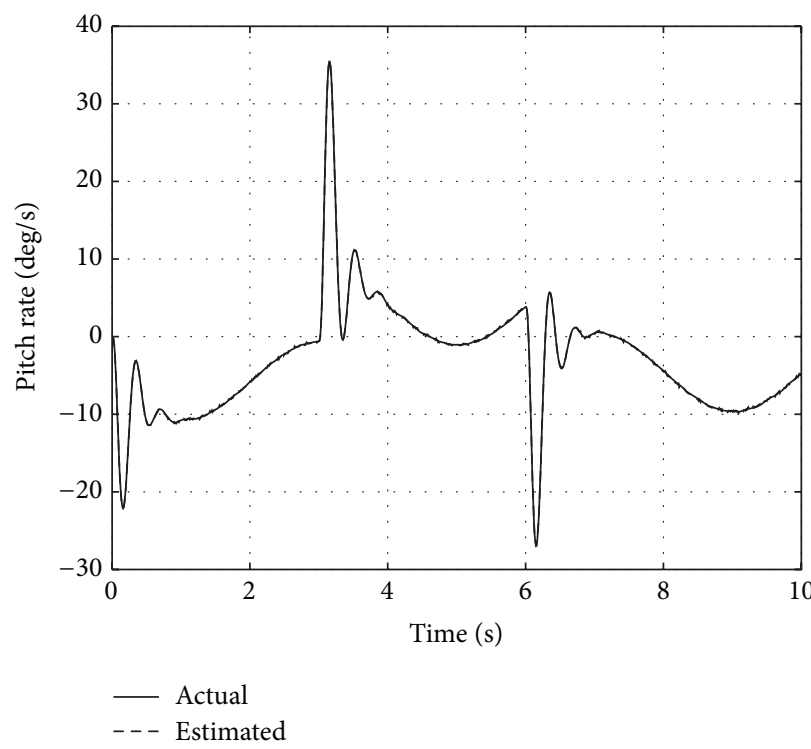

(b)

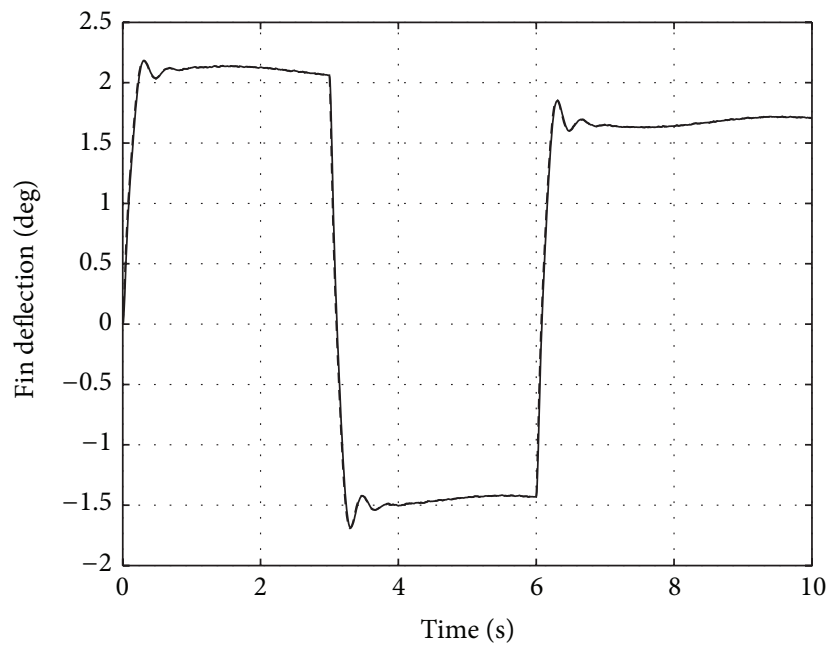

- Actual

- - - Estimated

(c)

FIGURE 7: Performance of GESO in state estimation with parametric perturbation in output channel.

(12) and (17) for plant (1)-(2) without any uncertainty and the results are presented in Figure 2. From the figures, it can be observed that the performance of both controllers is identical.

Next, simulations are carried out by introducing parametric uncertainties, unmodeled dynamics, measurement noise, and external disturbances. Parametric perturbations are introduced in aerodynamic derivatives except the one that appears in output channel (i.e., in $z_{\alpha}$ and $z_{\delta}$ ) for the reason explained later. To this end, uncertainties of $-20 \%$ in $m_{\alpha}, m_{q}$, and $m_{\delta}$ of their respective nominal values are introduced in the plant dynamics. The accelerometer and rate gyro measurements are assumed to be corrupted by white noise of standard deviation of $0.01 \mathrm{~m} / \mathrm{s}^{2}$ and $0.0667 \mathrm{deg} / \mathrm{s}$, respectively [47]. Further, time-varying external disturbance $d_{\alpha}^{\prime}=5 \sin (2 \pi 0.25 t) \mathrm{deg} / \mathrm{s}$ is introduced in $\alpha$ channel. Lastly, unmodeled dynamics of first order with time constant of 0.01 are used in series of the plant. For the purpose of comparison of actual uncertainty with its estimated value, actual uncertainty is computed from (1) as $d_{\alpha}=\dot{\alpha}-z_{\alpha} \alpha-z_{\delta} \delta$ and $d_{q}=\dot{q}-m_{\alpha} \alpha-m_{q} q-m_{\delta} \delta$. With these data, simulations are carried out considering the same reference input and the results are presented in Figures 3-5. In Figure 3, the output tracking performance of the predictive and GESO based predictive controller is presented. The commanded and achieved acceleration histories are shown in Figure 3(a), from where it can be seen that the GESO based predictive controller has offered accurate tracking of the output notwithstanding the uncertainties and disturbances. The corresponding control input histories are shown in Figure 3(b). The state and uncertainty estimation performance of GESO is given in 


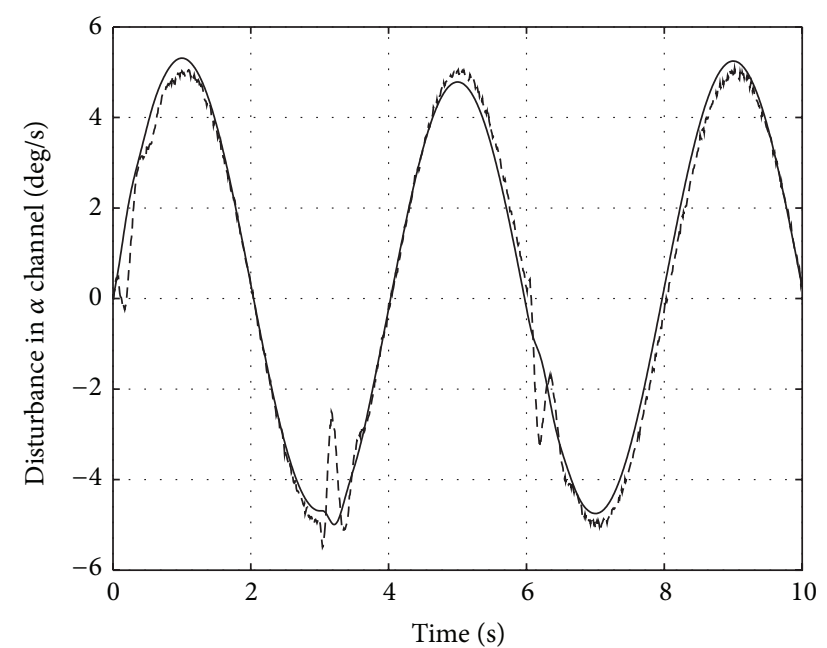

- Actual

- - Estimated

(a)

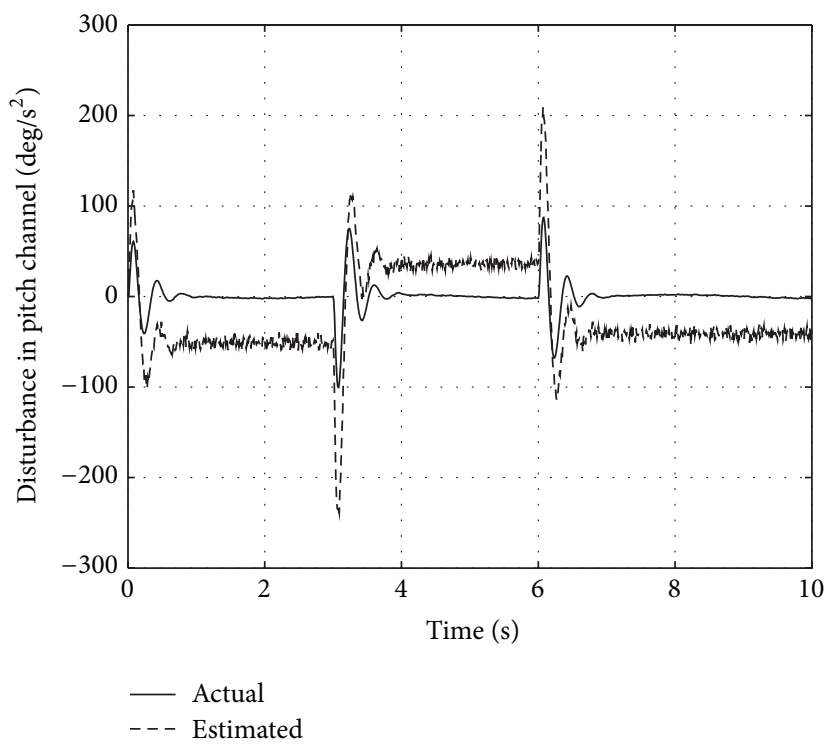

(b)

FIGURE 8: Performance of GESO in uncertainty estimation with parametric perturbation in output channel.

Figures 4 and 5, from where it can be seen that the GESO has estimated the states as well as the uncertainties quite accurately.

Lastly, simulations are carried out by including parametric uncertainties of $+5 \%$ in $z_{\alpha}$ in addition to the aforementioned uncertainties, disturbances, unmodeled dynamics, and measurement noise and the results are presented in Figures 6-8. Comparing the state and uncertainty estimation results of Figures 7-8 with the results of Figures 4-5, it can be observed that the estimation accuracy is marginally degraded. Introduction of uncertainty in the derivatives appearing in the output channel leads to uncertain $C_{m}$ in (14). Since the GESO formulation caters for uncertainties in state equation only, the state and uncertainty estimation in the event of uncertainty in output matrix leads to marginally degraded performance. While addressing the issue of uncertainties in output equation in GESO framework can form a scope for further research, the tracking performance of the proposed GESO based predictive controller in the presence of uncertainties in output channel is quite satisfactory as can be seen from Figure 6 .

In the simulation results presented in this section, it is important to note that the predictive controller of (12) has been implemented using exact values of the states, whereas the GESO based controller (17) uses the GESO estimated states. It is logical that when the predictive controller (12) is implemented in conjunction with a separately designed state observer, the performance would not be the same and is likely to degrade further in comparison with the GESO based predictive controller.

\section{Conclusion}

In this work, generalized extended state observer (GESO) robustified continuous time predictive control based acceleration tracking controller for pitch autopilot design of a cruciform, roll position stabilized, tail controlled tactical missile is proposed. The predictive control yields the feedback control gain and disturbance compensation gain simultaneously avoiding separate design procedures. Closed loop stability for the proposed design is established. The effectiveness of the GESO in estimation of the states and uncertainty and tracking of the commanded acceleration using the GESO based predictive controller is demonstrated through simulation. The results show that the GESO robustifies the predictive controller effectively leading to satisfactory tracking of the desired command in spite of the considered parametric uncertainties, unmodeled dynamics, external disturbance, and measurement noise. Lastly, as a further scope of research, the issue of uncertainty in output equation in GESO is highlighted.

\section{Competing Interests}

The authors declare that they have no competing interests.

\section{References}

[1] P. Garnell, Guided Weapon Control Systems, Pergamon Press, Oxford, UK, 2nd edition, 1980.

[2] M. P. Horton, "Autopilots for tactical missiles: an overview," Proceedings of the Institution of Mechanical Engineers. Part I, Journal of Systems and Control Engineering, vol. 209, no. 2, pp. 127-139, 1995.

[3] P. Zarchan, Tactical and Strategic Missile Guidance, vol. 199 of Progress in Astronautics and Aeronautics, American Institute of Aeronautics and Astronautics, Reston, Va, USA, 4th edition, 2002. 
[4] R. A. Nichols, R. T. Reichert, and W. J. Rugh, "Gain scheduling for H-infinity controllers: a flight control example," IEEE Transactions on Control Systems Technology, vol. 1, no. 2, pp. 69-78, 1993.

[5] R. T. Reichert, "Robust autopilot design using $\mu$-synthesis," in Proceedings of the American Control Conference, pp. 2368-2373, May 1990.

[6] S. Bennani, D. M. C. Willemsen, and C. W. Scherer, "Robust control of linear parametrically varying systems with bounded rates," Journal of Guidance, Control, and Dynamics, vol. 21, no. 6, pp. 916-922, 1998.

[7] A. R. Mehrabian and J. Roshanian, "Skid-to-turn missile autopilot design using scheduled eigenstructure assignment technique," Proceedings of the Institution of Mechanical Engineers, Part G: Journal of Aerospace Engineering, vol. 220, no. 3, pp. 225239, 2006.

[8] D. G. Benshabat and Y. Chait, "Application of quantitative feedback theory to a class of missiles," Journal of Guidance, Control, and Dynamics, vol. 16, no. 1, pp. 47-52, 1993.

[9] J. J. Zhu and M. C. Mickle, "Missile autopilot design using a new linear time-varying control technique," Journal of Guidance, Control, and Dynamics, vol. 20, no. 1, pp. 150-157, 1997.

[10] M. Tahk, M. M. Briggs, and P. K. A. Menon, "Applications of plant inversion via state feedback to missile autopilot design," in Proceedings of the 27th IEEE Conference on Decision and Control, pp. 730-735, Austin,Tex, USA, December 1988.

[11] V. Upreti, S. E. Talole, and S. B. Phadke, "Predictive estimation and control based missile autopilot design," in Proceedings of the AIAA Guidance, Navigation, and Control Conference and Exhibit (AIAA '04), pp. 2854-2863, Providence, RI, USA, August 2004.

[12] D. Chwa and J. Y. Choi, "Observer-based control for tailcontrolled skid-to-turn missiles using a parametric affine model," IEEE Transactions on Control Systems Technology, vol. 12, no. 1, pp. 167-175, 2004.

[13] T. Li, S. Zhang, H. Yang, Y. Zhang, and L. Zhang, "Robust missile longitudinal autopilot design based on equivalent-inputdisturbance and generalized extended state observer approach," Proceedings of the Institution of Mechanical Engineers, Part G: Journal of Aerospace Engineering, vol. 229, no. 6, pp. 1025-1042, 2015.

[14] S.-H. Kim, Y.-S. Kim, and C. Song, "A robust adaptive nonlinear control approach to missile autopilot design," Control Engineering Practice, vol. 12, no. 2, pp. 149-154, 2004.

[15] W.-H. Chen, "Nonlinear disturbance observer-enhanced dynamic inversion control of missiles," Journal of Guidance, Control, and Dynamics, vol. 26, no. 1, pp. 161-166, 2003.

[16] A. J. Calise, M. Sharma, and J. E. Corban, "Adaptive autopilot design for guided munitions," Journal of Guidance, Control, and Dynamics, vol. 23, no. 5, pp. 837-843, 2000.

[17] A. A. Godbole, T. R. Libin, and S. E. Talole, "Extended state observer-based robust pitch autopilot design for tactical missiles," Proceedings of the Institution of Mechanical Engineers, Part G, vol. 226, no. 12, pp. 1482-1501, 2012.

[18] P. Lu, "Nonlinear predictive controllers for continuous systems," Journal of Guidance, Control, and Dynamics, vol. 17, no. 3, pp. 553-560, 1994.

[19] P. Lu, "Optimal predictive control of continuous nonlinear systems," International Journal of Control, vol. 62, no. 3, pp. 633649, 1995.
[20] S. Li, J. Yang, W.-H. Chen, and X. Chen, "Generalized extended state observer based control for systems with mismatched uncertainties," IEEE Transactions on Industrial Electronics, vol. 59, no. 12, pp. 4792-4802, 2012.

[21] S. Li, J. Yang, W.-H. Chen, and X. Chen, Disturbance ObserverBased Control: Methods and Applications, CRC Press, Boca Raton, Fla, USA, 2014.

[22] P. Lu and B. L. Pierson, "Aircraft terrain following based on a nonlinear continuous predictive control approach," Journal of Guidance, Control, and Dynamics, vol. 18, no. 4, pp. 817-823, 1995.

[23] P. Lu, "Entry guidance and trajectory control for reusable launch vehicle," Journal of Guidance, Control, and Dynamics, vol. 20, no. 1, pp. 143-149, 1997.

[24] S. N. Singh, M. Steinberg, and R. D. DiGirolamo, "Nonlinear predictive control of feedback linearizable systems and flight control system design," Journal of Guidance, Control, and Dynamics, vol. 18, no. 5, pp. 1023-1028, 1995.

[25] S. E. Talole and R. N. Banavar, "Proportional navigation through predictive control," Journal of Guidance, Control, and Dynamics, vol. 21, no. 6, pp. 1004-1006, 1998.

[26] S. E. Talole, S. B. Phadke, and R. K. Singh, "Predictive homing guidance using time delay control," in Proceedings of the AIAA Guidance, Navigation, and Control Conference and Exhibit, AIAA 2005-6159, pp. 3122-3130, San Francisco, Calif, USA, August 2005.

[27] R. A. Hull, D. Schumacher, and Z. Qu, "Design and evaluation of robust nonlinear missile autopilots from a performance perspective," in Proceedings of the American Control Conference, pp. 189-193, Seattle, Wash, USA, June 1995.

[28] B. Panchal, J. P. Kolhe, and S. E. Talole, "Robust predictive control of a class of nonlinear systems," Journal of Guidance, Control, and Dynamics, vol. 37, no. 5, pp. 1437-1445, 2014.

[29] R. H. C. Takahashi and P. L. D. Peres, "Unknown input observers for uncertain systems: a unifying approach," European Journal of Control, vol. 5, no. 2-4, pp. 261-275, 1999.

[30] E. Schrijver and J. van Dijk, "Disturbance observers for rigid mechanical systems: equivalence, stability, and design," Journal of Dynamic Systems, Measurement, and Control, vol. 124, no. 4, pp. 539-548, 2002.

[31] S. E. Talole, J. Benito, and K. D. Mease, "Sliding mode observer for drag tracking in entry guidance," in Proceedings of the AIAA Guidance, Navigation, and Control Conference and Exhibit, AIAA 2007-6851, Hilton Head, SC, USA, August 2007.

[32] J.-H. She, M. Fang, Y. Ohyama, H. Hashimoto, and M. Wu, "Improving disturbance-rejection performance based on an equivalent-input-disturbance approach," IEEE Transactions on Industrial Electronics, vol. 55, no. 1, pp. 380-389, 2008.

[33] K. Youcef-Toumi and O. Ito, "A time delay controller for systems with unknown dynamics," Journal of Dynamic Systems, Measurement and Control, vol. 112, no. 1, pp. 133-142, 1990.

[34] Q.-C. Zhong and D. Rees, "Control of uncertain LTI systems based on an uncertainty and disturbance estimator," Transactions of the ASME, Journal of Dynamic Systems, Measurement and Control, vol. 126, no. 4, pp. 905-910, 2004.

[35] S. E. Talole, N. K. Singh, and S. B. Phadke, "Robust input-output linearization using UDE," in Proceedings of the International Conference on Advances in Control and Optimization of Dynamical Systems (ACODS '07), pp. 79-82, Bangalore, India, February 2007. 
[36] J. Han, "From PID to active disturbance rejection control," IEEE Transactions on Industrial Electronics, vol. 56, no. 3, pp. 900906, 2009.

[37] W. Wang and Z. Gao, "A comparison study of advanced state observer design techniques," in Proceedings of the American Control Conference, pp. 4754-4759, Denver, Colo, USA, June 2003.

[38] Z. Gao, Y. Huang, and J. Han, "An alternative paradigm for control system design," in Proceedings of the 40th IEEE Conference on Decision and Control (CDC'01), vol. 5, pp. 45784585, Orlando, Fla, USA, December 2001.

[39] A. A. Godbole, J. P. Kolhe, and S. E. Talole, "Performance analysis of generalized extended state observer in tackling sinusoidal disturbances," IEEE Transactions on Control Systems Technology, vol. 21, no. 6, pp. 2212-2223, 2013.

[40] J. Yao, Z. Jiao, and D. Ma, "Adaptive robust control of dc motors with extended state observer," IEEE Transactions on Industrial Electronics, vol. 61, no. 7, pp. 3630-3637, 2014.

[41] S. Li and Z. Liu, "Adaptive speed control for permanent-magnet synchronous motor system with variations of load inertia," IEEE Transactions on Industrial Electronics, vol. 56, no. 8, pp. 30503059, 2009.

[42] Y. Xia, Z. Zhu, M. Fu, and S. Wang, "Attitude tracking of rigid spacecraft with bounded disturbances," IEEE Transactions on Industrial Electronics, vol. 58, no. 2, pp. 647-659, 2011.

[43] S. E. Talole, J. P. Kolhe, and S. B. Phadke, "Extended-stateobserver-based control of flexible-joint system with experimental validation," IEEE Transactions on Industrial Electronics, vol. 57, no. 4, pp. 1411-1419, 2010.

[44] J. Yao, Z. Jiao, and D. Ma, "Extended-state-observer-based output feedback nonlinear robust control of hydraulic systems with backstepping," IEEE Transactions on Industrial Electronics, vol. 61, no. 11, pp. 6285-6293, 2014.

[45] Z. Zhu, D. Xu, J. Liu, and Y. Xia, "Missile guidance law based on extended state observer," IEEE Transactions on Industrial Electronics, vol. 60, no. 12, pp. 5882-5891, 2013.

[46] S. E. Talole, A. A. Godbole, J. P. Kolhe, and S. B. Phadke, "Robust roll autopilot design for tactical missiles," Journal of Guidance, Control, and Dynamics, vol. 34, no. 1, pp. 107-117, 2011.

[47] P. Gurfil, "Zero-miss-distance guidance law based on line-ofsight rate measurement only," Control Engineering Practice, vol. 11, no. 7, pp. 819-832, 2003. 


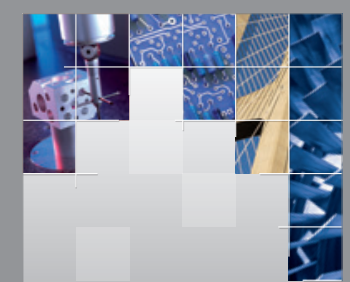

\section{Enfincering}
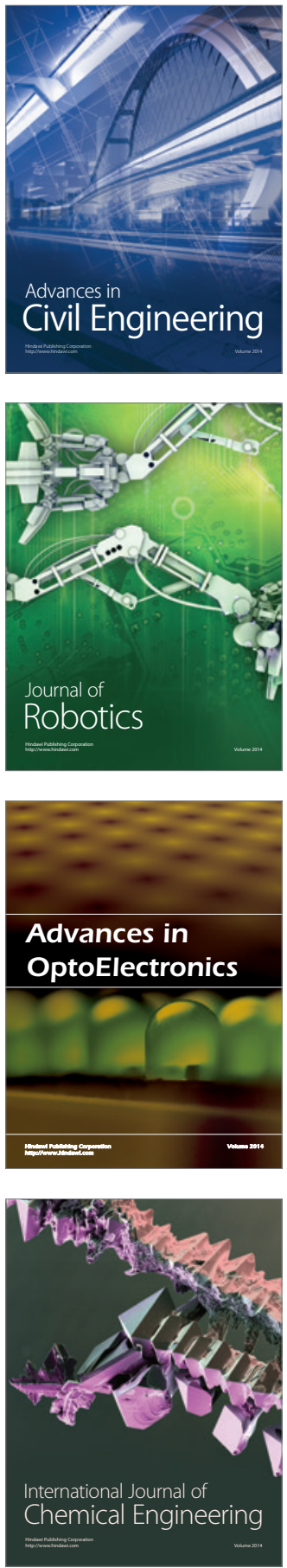

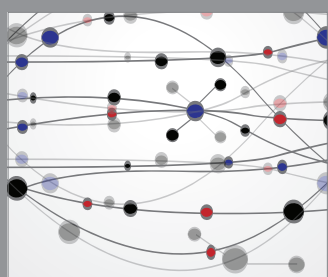

The Scientific World Journal

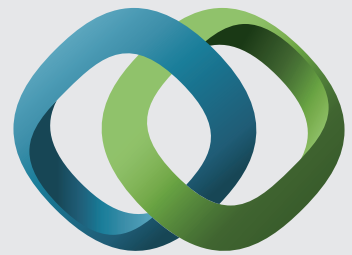

\section{Hindawi}

Submit your manuscripts at

http://www.hindawi.com
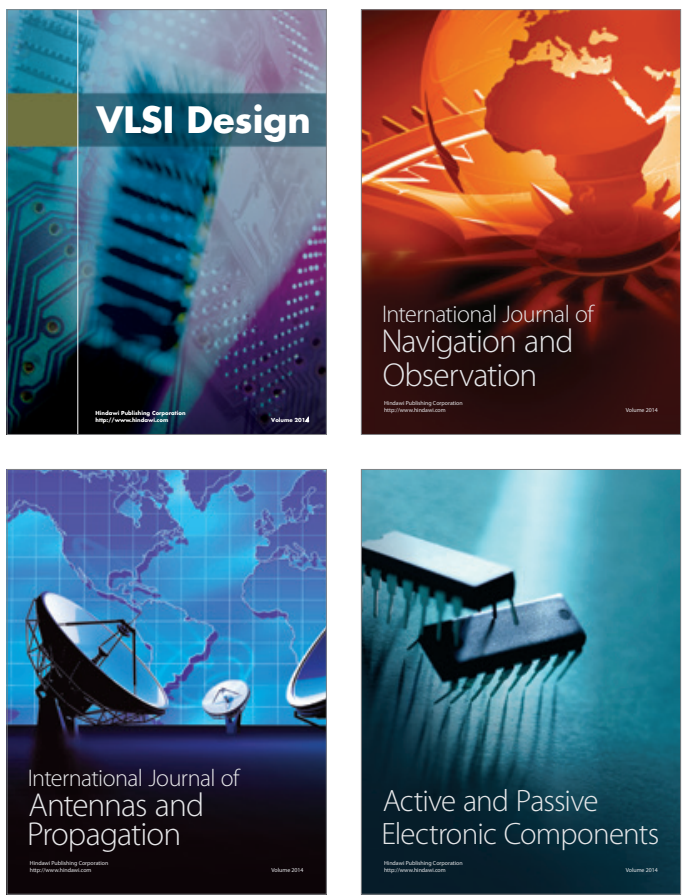
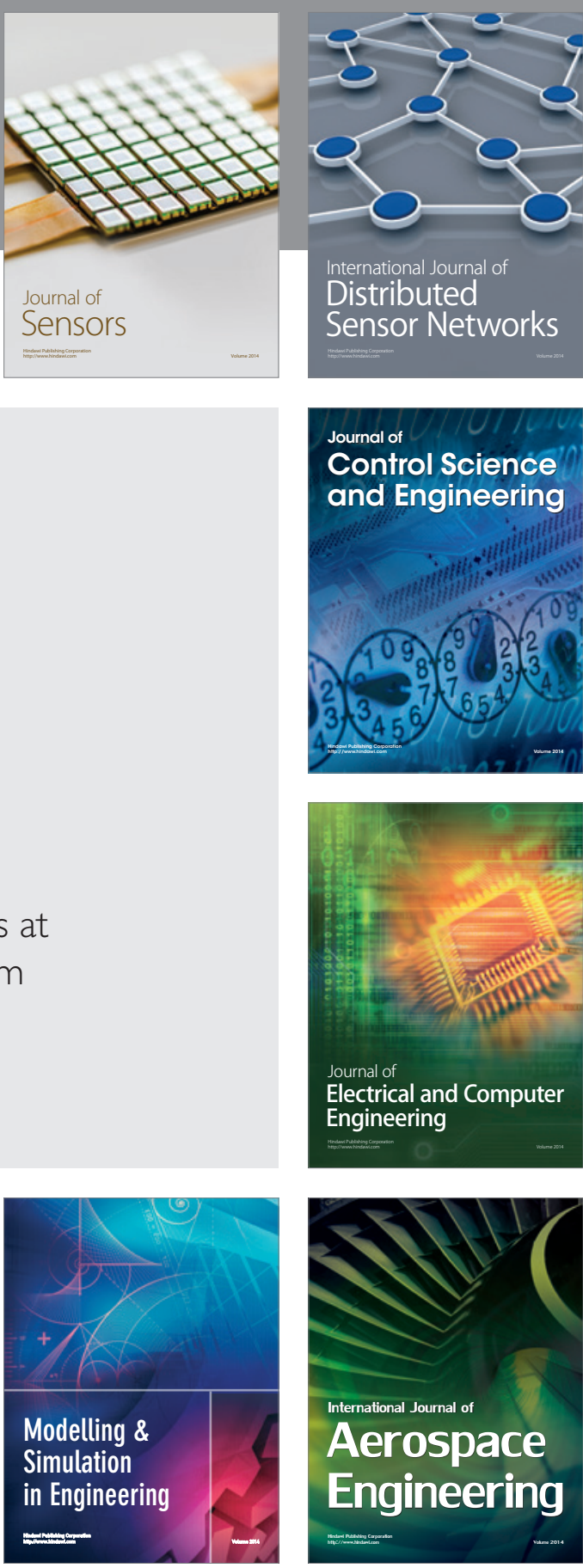

International Journal of

Distributed

Sensor Networks

Journal of

Control Science

and Engineering
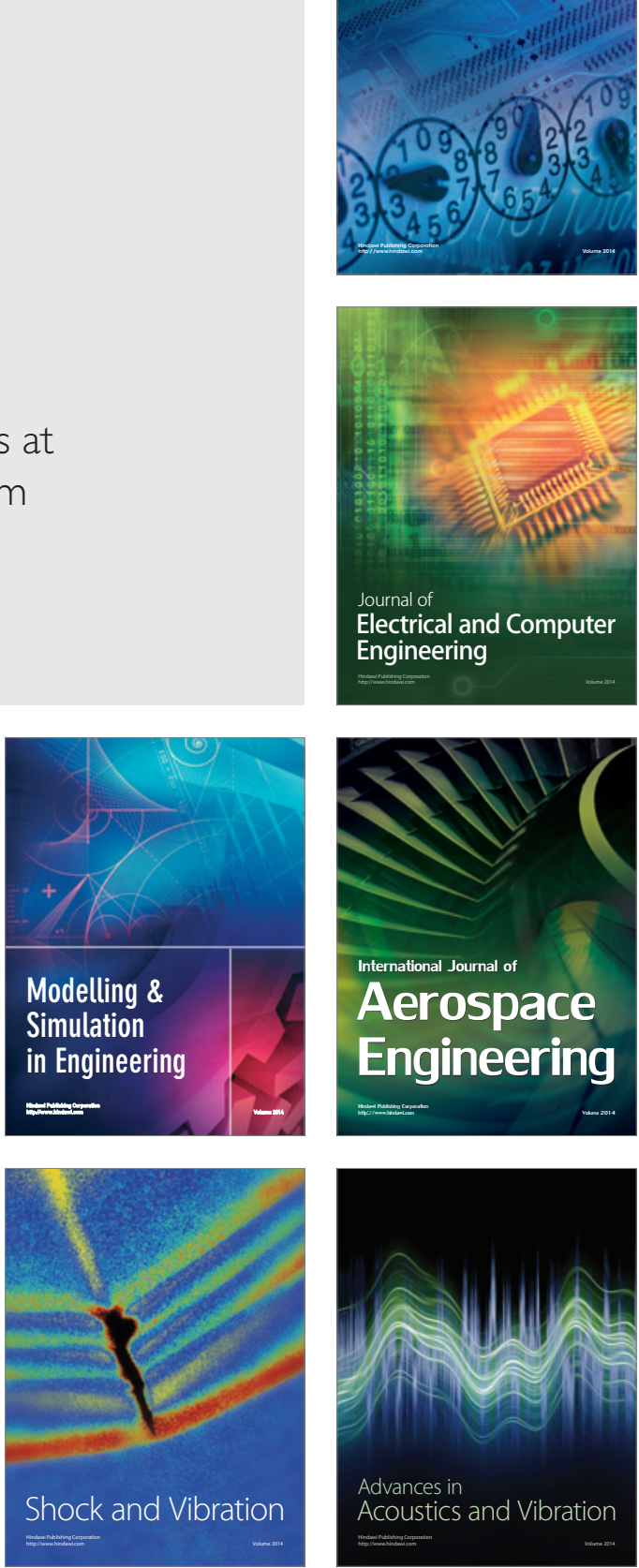